\title{
Biphasic Levodopa-Induced Freezing of Gait in Parkinson's Disease
}

\author{
Jorik Nonnekes $^{\mathrm{a}, \mathrm{b}, *}$ and Bastiaan R. Bloem ${ }^{\mathrm{c}}$ \\ ${ }^{\mathrm{a}}$ Radboud University Medical Centre, Donders Institute for Brain, Cognition and Behaviour, \\ Department of Rehabilitation, Center of Expertise for Parkinson \& Movement Disorders, \\ Nijmegen, The Netherlands \\ ${ }^{\mathrm{b}}$ Department of Rehabilitation, Sint Maartenskliniek, Nijmegen, The Netherlands \\ ${ }^{\mathrm{c}}$ Radboud University Medical Centre, Donders Institute for Brain, Cognition and Behaviour, \\ Department of Neurology, Center of Expertise for Parkinson \& Movement Disorders, Nijmegen, \\ The Netherlands
}

Accepted 19 April 2020

\begin{abstract}
The relation between freezing of gait in Parkinson's disease and levodopa is complex. Here, we describe a new phenotype of freezing of gait, namely levodopa-induced freezing of gait with a biphasic pattern. Our observation supports the idea that freezing of gait might emerge because of a mismatch between cognitive/limbic loops and motor loops involved in gait control. Moreover, it underscores the importance of assessing the influence of dopaminergic medication in daily clinical practice, including objective assessment in all three dopaminergic states. The possibility of biphasic freezing will only emerge after such a comprehensive evaluation, and will have immediate therapeutic consequences.
\end{abstract}

Keywords: Gait, freezing of gait, levodopa, Parkinson's disease, parkinsonism, biphasic

Freezing of gait is common and disabling in people with Parkinson's disease, and is characterized clinically by an episodic inability to produce effective forward stepping [1]. The relation between freezing of gait and levodopa is complex [2], and three main types of responses to levodopa have been identified. The most common treatment response is freezing of gait that improves with levodopa treatment; this is most typically present in early disease stages. The second type is levodopa-resistant freezing of gait, which usually emerges with disease progression, either because of development of non-dopaminergic lesions outside the substantia nigra, or because factors such as constipation or dose-limiting dyskinesias

\footnotetext{
*Correspondence to: Jorik Nonnekes, MD, PhD, Radboud University Medical Center, PO Box 9101, 6500 HB Nijmegen, The Netherlands. E-mail: jorik.nonnekes@ radboudumc.nl.
}

hamper optimal delivery of levodopa therapy [3]. The third and final response type is levodopa-induced freezing of gait, where freezing occurs predominantly in the dopaminergic on-state, and less so-or not at all-during the dopaminergic off-state [4]. Here, we describe-for the first time-a fourth response type that had hitherto only been associated with dyskinesias, namely levodopa-induced freezing of gait with a biphasic pattern.

A 70-year-old man was referred to our outpatient clinic because of disabling freezing of gait. Parkinson's disease had been diagnosed 5 years earlier. He was treated with levodopa/carbidopa 100/25 mg five times daily. He rarely experienced freezing of gait in the early morning, before the first dose of dopaminergic medication. During these early morning times, he described his gait as slow and shuffling, but distinctly without any gluing or sudden motor 
blocks. He similarly shuffled when visiting the bathroom at night, but never experienced any freezing of gait at such times. After the first gift of levodopa/carbidopa in the morning, episodes of freezing of gait would emerge, during which he experienced an inability to initiate walking despite the intention and desire to do so. He further experienced that after taking another tablet of levodopa/carbidopa shortly after the first intake, freezing of gait disappeared. To objectify this pattern, we invited him to visit our hospital in a practically defined dopaminergic off-state ( $>12$ hours after the last intake of levodopa). During a videotaped and standardised gait assessment (including rising from sit-to-stance, and a walking trajectory of approximately 10 meters, including a 180- and 360-degrees turn), we observed a hypokinetic gait pattern, but no freezing of gait occurred (Video 1). Fifteen minutes after intake of a single dose of levodopa/carbidopa 100/25 mg, freezing of gait with attempted stepping emerged, specifically during gait initiation and when approaching the chair (Video 2). He reported feeling capable of walking, but being episodically unable to do so. After intake of an extra dose of levodopa/carbidopa 200/50 mg, he walked faster with larger steps, and no freezing of gait was seen (Video 3). Based on this assessment, his maintenance dose of dopaminergic medication was gradually increased (ultimately to levodopa/carbidopa $150 / 37.5 \mathrm{mg}$ five times daily), and freezing of gait in daily life decreased significantly and satisfactorily. The higher levodopa dose did come with a worsening of dyskinesias, which we later tried to suppress with amantadine $100 \mathrm{mg}$ twice daily. These dyskinesias had a typical peak dose pattern, and not the biphasic pattern observed for the freezing of gait.

A biphasic or square wave patterned dopa is wellknown for levodopa-induced dyskinesias [5], but levodopa-induced freezing of gait with a biphasic pattern has not been described before. The implications of this finding are twofold. First, our observations further underscore the complex relation between levodopa and freezing of gait. The existence of levodopa-induced biphasic freezing provides additional support for the idea that freezing of gait might emerge because of a mismatch between cognitive/limbic loops and motor loops involved in gait control [6]. We recently introduced a novel framework better understand the complex role of levodopa in the occurrence of freezing of gait [6]. Specifically, we emphasised that gait control depends on input from motor loops, but also on cognitive and limbic inputs [7]. In patients with Parkinson's disease, dopaminergic motor loops are denervated to a greater extent than dopaminergic limbic and cortical loops, and this mismatch may become even larger due to maladaptive plasticity induced by long-term pulsatile levodopa treatment, which might disproportionately affect the motor loops. Freezing of gait may thus emerge when there is a mismatch between activated cognitive and limbic loops on the one hand (causing a desire to walk and a feeling of being capable to do so), and faulty motor loops on the other hand (causing an inability to actually initiate the desired step).
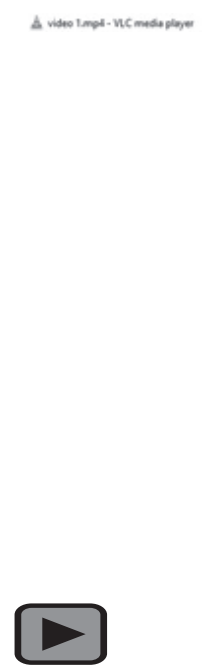

Video 1. Gait assessment in a 70-year-old patient with idiopathic Parkinson's disease, in the dopaminergic off-state ( $>12$ hours after the last medication intake). There is a hypokinetic gait pattern (also when starting to walk), but without freezing of gait. 


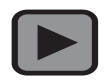

Video 2. Gait assessment 15 minutes after intake of levodopa/carbidopa 100/25 mg. Freezing of gait with attempted stepping emerges during gait initiation and when approaching the chair.

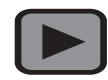

Video 3. Gait assessment 15 minutes after intake of levodopa/carbidopa 200/50 mg. Compared to the dopaminergic off-state, there is a reduction of the hypokinetic gait features. Freezing of gait does not occur.

In our patient with levodopa-induced biphasic freezing of gait, such a mismatch might also have arisen after intake of low doses of levodopa that successfully stimulated cognitive and limbic functioning, but that were unable to exceed the dopaminergic thresholds within the motor loops. Yet higher levels of dopaminergic medication could exceed these motor thresholds, and suppress further freezing of gait.

Second, in daily clinical practice, the influence of dopaminergic medication should be evaluated carefully in people with freezing of gait, including objective and preferably videotaped assessment in all three dopaminergic states (off state, after low dose levodopa, and after a suprathreshold levodopa dose) [8]. We initially considered the possibility of levodopa-induced freezing in our patient, but the possibility of biphasic freezing only emerged after such a comprehensive evaluation, and this had immediate therapeutic consequences (further increasing the dose of levodopa). Further work is needed to determine how common this biphasic type of freezing occurs in daily clinical practice. It will also be interesting to see if such biphasic freezing is always uncoupled from biphasic dyskinesias as was the case in 
our patient, or whether these can co-occur within the same individuals.

\section{ACKNOWLEDGMENTS}

The Centre of Expertise for Parkinson \& Movement Disorders was supported by a centre of excellence grant by the Parkinson Foundation. Jorik Nonnekes was supported by a ZonMW Off Road grant.

\section{CONFLICT OF INTEREST}

The authors report no conflict of interest.

\section{REFERENCES}

[1] Weiss D, Schoellmann A, Fox MD, Bohnen NI, Factor SA, Nieuwboer A, Hallett M, Lewis SJG (2020) Freezing of gait: Understanding the complexity of an enigmatic phenomenon. Brain 143, 14-30.
[2] Nutt JG, Bloem BR, Giladi N, Hallett M, Horak FB, Nieuwboer A (2011) Freezing of gait: Moving forward on a mysterious clinical phenomenon. Lancet Neurol 10, 734-744.

[3] Nonnekes J, Timmer MH, de Vries NM, Rascol O, Helmich RC, Bloem BR (2016) Unmasking levodopa resistance in Parkinson's disease. Mov Disord 31, 1602-1609.

[4] Espay AJ, Fasano A, van Nuenen BF, Payne MM, Snijders AH, Bloem BR (2012) "On" state freezing of gait in Parkinson disease: A paradoxical levodopa-induced complication. Neurology 78, 454-457.

[5] Calabresi P, Di Filippo M, Ghiglieri V, Tambasco N, Picconi B (2010) Levodopa-induced dyskinesias in patients with Parkinson's disease: Filling the bench-to-bedside gap. Lancet Neurol 9, 1106-1117.

[6] Nonnekes J, Bereau M, Bloem BR (2019) Freezing of gait and its levodopa paradox. JAMA Neurol. doi: 10.1001/jamaneurol.2019.4006.

[7] Lewis SJ, Barker RA (2009) A pathophysiological model of freezing of gait in Parkinson's disease. Parkinsonism Relat Disord 15, 333-338.

[8] Nonnekes J, Snijders AH, Nutt JG, Deuschl G, Giladi N, Bloem BR (2015) Freezing of gait: A practical approach to management. Lancet Neurol 14, 768-778. 